\title{
A Novel Evolutionary Algorithm for Capacitor Placement in Distribution Systems
}

\author{
Ji-Pyng Chiou and Chung-Fu Chang
}

\begin{abstract}
This paper uses an effective method, CODEQ method, with integer programming for solving the capacitor placement problems in distribution systems. Different from the original differential evolution (DE), the concepts of chaotic search, opposition-based learning, and quantum mechanics are used in the CODEQ method to overcome the drawback of selection of the crossover factor and scaling factor used in the original DE method. One benchmark function and one 9-bus system from the literature are used to compare the performance of the CODEQ method with the DE, and simulated annealing (SA). Numerical results show that the performance of the CODEQ method is better than the other methods. Also, the CODEQ method used in 9-bus system is superior to some other methods in terms of solution power loss and costs.
\end{abstract}

Index Terms - Capacitor Placement. Chaotic Search. CODEQ. Opposition-based Learning. Quantum Mechanics.

\section{INTRODUCTION}

$\mathrm{C}$ apacitors are widely installed in distribution systems for reactive power compensation to achieve power and energy reduction, voltage regulation and system capacity release. And, the installation of shunt capacitors in primary distribution systems can also effectively reduce peak power and energy losses. The extent of these benefits depends greatly on how the capacitors are placed on the system, namely on the location and size of the added capacitors [1,2]. The objective in the capacitor placement problem is to minimize the annual cost of the system, subject to operating constraints under a certain load pattern. Grainger et al. [3-5] proposed the concept that the size of capacitor banks was considered as a continuous variable. Bala et al. [6] presented a sensitivity-based method to solve the optimal capacitor placement problem. Using genetic algorithm (GA) to select capacitors for radial distribution systems was proposed in [7]. In the above-mentioned methods, the capacitors were often assumed as continuous variables, in which cost is proportionate to the capacitor size. However, commercially available capacitors are discrete. Selecting integer capacitor sizes closest to the optimal values found by the

Manuscript received October 28, 2013.

J. P. Chiou is with the Department of Electrical Engineering, Ming Chi University of Technology, New Taipei City, Taiwan (phone: +886-2-2908-9899\#4804; fax:+886-2-2908-4507; e-mail: jipyng@ mail.mcut.edu.tw).

C. F. Chang is with the Department of Electrical Engineering, WuFeng University, Chiayi County, Taiwan (e-mail: cfchang@wfu.edu.tw). continuous variable approach may not guarantee an optimal solution [8]. Therefore the optimal capacitor placement should be viewed as an integer-programming problem, and discrete capacitors will be considered in this paper. The teaching learning based optimization (TLBO) approach to minimize power loss and energy cost by optimal placement of capacitors in radial distribution systems [9]. Muthu Kumar et al. [10] proposed an opposition based differential evolution (ODE) method for a distribution system reconfiguration to operate the system at minimum cost and at the same time improves the system reliability and security. Olamael et al. [11] proposed a new adaptive modified firefly algorithm to solve optimal capacitor placement problem.

Differential evolution (DE) as developed by Stron and Price [8] is one of the best Evolutionary algorithms (EAs), and has proven to be a promising candidate to solve real valued optimization problems [12]. This method also turned out to be one of the best genetic algorithms for solving the real-valued test function suite of the first International Contest on Evolutionary Computation, which was held in Nagoya in 1996. $\mathrm{DE}$ is a stochastic search and optimization method. The fittest of an offspring competes one-to-one with that of the corresponding parent, which is different from the other EAs. This one-to-one competition gives rise to a faster convergence rate. However, this faster convergence also leads to a higher probability of obtaining a local optimum because the diversity of the population descends faster during the solution process. To overcome this drawback, the parameters selection is very important for the DE method. However, the parameters selection is more sensitive with the problem. For example, a fixed scaling factor is used in DE. Using a smaller scaling factor, DE becomes increasingly robust. However, much computational time should be expanded to evaluate the objective function. DE with a larger scaling factor should result generally falls into local solution or misconvergence. Lin et al. [13] used a random number that its value is between zero and one as a scaling factor. However, a random scaling factor could not guarantee the fast convergence. So, Omran et al. [14] presented an effective method, CODEQ method, to overcome the drawback of the parameters selection problem. Only two parameters, population size and the maximum iteration, are necessary for the CODEQ method.

In this study, a CODEQ method [14-16] with integer programming for solving the optimal capacitor placement of 
distribution systems is proposed. Here, the concepts of chaotic search, opposition-based learning, and quantum mechanics are used in the CODEQ method to overcome the drawback of selection of the crossover factor and scaling factor used in the original DE method. Optimal capacitor placement is a combinatorial optimization problem that is commonly solved by employing mathematical programming techniques. However, in those methods, the capacitors are often assumed as continuous variables in which cost is proportionate to the capacitor size. Selecting integer capacitor sizes closet to the optimal values found by the continuous variable approach does not guarantee an optimal solution. Therefore, the optimal capacitor placement should be viewed as an integer-variable problem. The CODEQ method can be used to solve the integer-variable problems effectively. To illustrate the convergence property of the proposed method, one benchmark function and one 9-bus system from the literature are solved respectively by the proposed method, DE, and SA. From the computational results, it is observed that the convergence property of the CODEQ method is better than that of the other methods.

\section{Problem Formula}

The mathematical model of the optimal capacitor placement of distribution systems can be expressed as follows:

$$
\min \operatorname{COST}
$$

Subject to

$$
V_{\min } \leq\left|V_{i}\right| \leq V_{\max }
$$

Where $\left|V_{i}\right|$ is the voltage magnitude of bus $i, V_{\min }$ and $V_{\max }$ are the minimum and maximum voltage limits, respectively.

The objective function COST in (1) is an overall cost relating to power loss and capacitor placement. The voltage magnitude at each bus must be maintained between its minimum and maximum voltage limits. To avoid the complex iteration process for power flow analysis, a set of simplified feeder-line flow formulations is applied. Considering the single-line diagram depicted in Fig.1, the following set of recursive equations is used for power flow computation [17].

$$
\begin{aligned}
& P_{i+1}=P_{i}-P_{L i+1}-R_{i, i+1} \cdot\left(P_{i}^{2}+Q_{i}^{2}\right) /\left|V_{i}\right|^{2} \\
& Q_{i+1}=Q_{i}-Q_{L i+1}-X_{i, i+1} \cdot\left(P_{i}^{2}+Q_{i}^{2}\right) /\left|V_{i}\right|^{2} \\
& \left|V_{i+1}\right|^{2}=\left|V_{i}\right|^{2}-2\left(R_{i, i+1} \cdot P_{i}+X_{i, i+1} \cdot Q_{i}\right)+\left(R_{i, i+1}^{2}+X_{i, i+1}^{2}\right) \frac{\left(P_{i}^{2}+Q_{i}^{2}\right)}{\left|V_{i}\right|^{2}}
\end{aligned}
$$

Where $P_{i}$ and $Q_{i}$ are the real and reactive powers flowing out of bus $i$, and $P_{L i}$ and $Q_{L i}$ are the real and reactive load powers at bus $i$. The resistance and reactance of the line section between buses $i$ and $i+1$ are denoted by $R_{i, i+1}$ and $X_{i, i+1}$, respectively.

The power loss of the line section connecting buses $i$ and $i+1$ may be computed as
$P_{\text {Loss }}(i, i+1)=R_{i, i+1} \cdot \frac{P_{i}^{2}+Q_{i}^{2}}{\left|V_{i}\right|^{2}}$

The total power loss of the feeder, $P_{T, \text { Loss }}$, may then be determined by summing up the losses of all line sections of the feeder. Which is given by

$P_{T, \text { Loss }}=\sum_{i=0}^{n-1} P_{\text {Loss }}(i, i+1)$

Considering the real-world capacitors, there exists a finite number of standard sizes which are integer multiples of the smallest size $Q_{0}^{C}$. Besides, the cost per kVAr varies from one size to another.

In general, capacitors of larger size have lower unit prices. The available capacitor size is usually limited to

$Q_{\max }^{C}=L Q_{0}^{C}$

Where $L$ is an integer. Therefore, for each installation location, there are $L$ capacitor sizes $\left\{Q_{0}^{C}, 2 Q_{0}^{C}, \ldots, L Q_{0}^{C}\right\}$ available. Given the annual unit capacitor installation cost for each compensated bus, the total cost due to capacitor placement and power loss change is written as

$\operatorname{COST}=K_{p} P_{T, \text { Loss }}+\sum_{i=1}^{n} K_{i}^{C} Q_{i}^{C}$

Where $K_{p}$ is the equivalent annual cost per unit of power loss in $\$ /(k W-$ year $)$, and here $\$$ is a fictional monetary unit. The constant $K_{i}$ is the annual unit capacitor installation cost. And, $i=1,2, \ldots, n$ are the indices of buses selected for compensation. The bus reactive compensation power is limited to

$Q_{i}^{C} \leq \sum_{i=1}^{n} Q_{L i}$

Where $Q_{i}^{C}$ and $Q_{L i}$ are the reactive power compensated at bus $i$ and the reactive load power in bus $i$, respectively.

\section{Codeq Method}

The main idea of the CODEQ method is to use the concepts of chaotic search, opposition-based learning, and quantum mechanics into the original DE method to overcome the drawback of selection of the crossover factor and scaling factor. The CODEQ method is briefly described in the following.

Step 1. Initialization

Input system data and generate the initial population. The initial population is chosen randomly and would attempt to cover the entire parameter space uniformly. The uniform probability distribution for all random variables as following is assumed

$\mathbf{Z}_{i}^{0}=\mathbf{Z}_{\text {min }}+\operatorname{round}\left(\sigma_{i} \cdot\left(\mathbf{Z}_{\max }-\mathbf{Z}_{\min }\right)\right), i=1, \ldots, N_{p}$

Where $\sigma_{i} \in(0,1]$ is a random number, and $\operatorname{round}(b)$ represented as the nearest integer for the real number $b$. The 
initial process can produce $N_{p}$ individuals of $\mathbf{Z}_{i}^{0}$ randomly.

Step 2. Mutation operation

The essential ingredient in the mutation operation is the difference vector. Each individual pair in a population at the G-th generation defines a difference vector $\mathbf{D}_{j k}$ as

$\mathbf{D}_{j k}=\mathbf{Z}_{j}^{G}-\mathbf{Z}_{k}^{G}$

The mutation process at the G-th generation begins by randomly selecting either two or four population individuals $\mathbf{Z}_{j}^{G}, \mathbf{Z}_{k}^{G}, \mathbf{Z}_{l}^{G}$ and $\mathbf{Z}_{m}^{G}$ for any $j, k, l$ and $m$. These four individuals are then combined to form a difference vector $\mathbf{D}_{j k l m}$ as

$$
\mathbf{D}_{j k l m}=\mathbf{D}_{j k}+\mathbf{D}_{l m}=\left(\mathbf{Z}_{j}^{G}-\mathbf{Z}_{k}^{G}\right)+\left(\mathbf{Z}_{l}^{G}-\mathbf{Z}_{m}^{G}\right)
$$

A mutate vector is then generated based on the present individual in the mutation process by

$\hat{\mathbf{Z}}_{i}^{G+1}=\mathbf{Z}_{p}^{G}+F \cdot \mathbf{D}_{j k l m}, i=1, \ldots, N_{p}$

However, the scaling factor value is depends on the problem. Different from the original DE algorithm, the concept of the quantum mechanics $[14,18]$ is used to generate the noisy replica from the parent individual in CODEQ algorithm as follows:

$\hat{\mathbf{Z}}_{i}^{G+1}=\mathbf{Z}_{i}^{G}+\operatorname{round}\left(\left(\mathbf{Z}_{i 1}^{G}-\mathbf{Z}_{i 2}^{G}\right) \cdot \ln \left(\frac{1}{u}\right)\right), i=1, \ldots, N_{p}, i 1 \neq i 2 \neq i$

Where $u \in(0,1]$ is a random number.

Step 3. Estimation and selection

$\mathbf{Z}_{i}^{G+1}=\arg \min \left\{f\left(\mathbf{Z}_{i}^{G}\right), f\left(\hat{\mathbf{Z}}_{i}^{G+1}\right)\right\}$

$\mathbf{Z}_{b}^{G+1}=\arg \min \left\{f\left(\mathbf{Z}_{i}^{G}\right)\right\}$

Where arg min means the argument of the minimum.

Step 4. Exclude operation if necessary

To increase the convergence of the CODEQ algorithm, the exclude operation is considered. First, a new individual is created as follows:

$\mathbf{Z}_{w}^{G+1}=\left\{\begin{array}{lr}\mathbf{Z}_{\min }+\mathbf{Z}_{\max }-\gamma \cdot \mathbf{Z}_{\text {worst }}^{G+1}, & \text { if } \delta \leq 0.5 \\ \mathbf{Z}_{\text {best }}^{G+1}+\left|\mathbf{Z}_{i 1}^{G+1}-\mathbf{Z}_{i 2}^{G+1}\right| \cdot\left(2 \cdot c^{G+1}-1\right), \text { otherwise }\end{array}\right.$

Where $\gamma$ and $\delta$ are randomly generated numbers uniformly distributed in the range of $(0,1) . \mathbf{Z}_{\text {worst }}^{G+1}$ and $\mathbf{Z}_{\text {best }}^{G+1}$ are the worst and best individual in the $(\mathrm{G}+1)$ th generation. $c^{G+1}$ is the chaotic variable defined as follow:

$c^{G+1}= \begin{cases}c^{G} / p, & \text { if } c^{G} \in(0, p) \\ \left(1-c^{G}\right) /(1-p), & \text { if } c^{G} \in[p, 1)\end{cases}$

Where $c^{0}$ and $p$ are initialized randomly within the interval $(0,1)$.

The worst individual in the G-th generation is replaced by the generated individual, if the fitness of the generated individual is better than that of worst individual in the G-th generation.

Step 5. Repeat step 2 to step 4 until the maximum iteration quantity or the desired fitness is accomplished.

\section{Application of the Proposed Method}

One benchmark function and one 9-bus system from the literature are investigated and the results are used to compare the performance of the CODEQ method with the DE, and SA methods. The FORTRAN SA [19] algorithm solver accessed from http://www-aig.jpl.nasa.gov/public/home /decoste/TMLS /NN/glopt/glopt.html\#sa codes, is used to solve the optimal capacitor placement problems. The SA solver recommended some setting factors for a user. For comparison, the SA package is rewritten using Matlab software.

Example 1: Let us consider the maximization problem is described by

$$
\max _{z_{1}, z_{2}} J\left(z_{1}, z_{2}\right)=21.5+z_{1} \sin \left(4 \pi z_{1}\right)+z_{2} \sin \left(20 \pi z_{2}\right)
$$

Where $-3 \leq z_{1} \leq 12.1$ and $4.1 \leq z_{2} \leq 5.8$. This problem has been solved by the simple genetic algorithm using the population size of 20 . The best result in generation 396 had the best objective function value of 38.827553 , as also shown in Michalewicez [20].

To verify the performance of the CODEQ method, the convergence property of the CODEQ method and the original DE method are compared via this example firstly. The population size $N_{p}=20$, scaling factor $F=0.1$, crossover factor $C_{R}=0.5$, and maximum iterations of 300 , are used in the DE method for solving this example. Six strategies of mutation operation are respectively used to solve this example. This solution of this example is repeatedly solved one hundred times. The largest and smallest values among the best solutions of the one hundred runs are respectively expressed in Table I. The average for the best solutions of the one hundred runs and the standard deviation with respect to the average are also shown in this table. A smaller standard deviation implies that almost all the best solutions are close the average best solution. From the Table I, the standard deviation for the CODEQ method is smaller than the other mutation strategy. That implies the convergence property of the CODEQ method is better than the original DE method. Five parameters including the population size, mutation operation, crossover factor, scaling factor, and the maximum iteration number must be set in the original DE method. But, only two parameters, population size and maximum iteration number, needs to set in the CODEQ method. The best solutions for these one hundred runs are compared to the best objective function value obtained by the simple genetic algorithm. The number of times that these best solutions were greater than 38.827553 is shown in Table I. From the Table I, the number of the successful runs that the best solutions were greater than 38.827553 is $10,2,6,65,70$, and 14 for six different strategies of mutation operation. The number of the successful runs that the best solutions were greater than 38.827553 is 55 in the CODEQ method. Only fourth and fifth mutation strategy is better than the CODEQ method. Table II lists the standard deviation values when the population size is reassigned to 10 and 5 to solve this example one hundred times, respectively. From the above discussion, the convergence property of the CODEQ method is better than the original DE 
method.

Example 2: The application example [17] is a $23-\mathrm{kV}$, 9 -section feeder system. Details of the feeder and the load characteristics are given by [17]. The equivalent annual cost per unit of power loss, $K_{p}$, is selected to be $\$ 168 /(k W-$ year $)$ and the limits on the bus voltages are

$V_{\min }=0.90$ p.u.

$V_{\text {max }}=1.10$ p.u.

Two cases are investigated.

Case 1: It is restricted that only 3 locations (buses 4, 5, and 9) are available for placement of capacitors. The setting-factors used in the CODEQ to solve this case are as follows. The population size, $N_{p}$, is set to 5 . The maximum generation is 500. These initial-setting factors for the DE method are the same as that for the CODEQ, except that the DE uses a scaling factor fixed to 0.6 , a crossover factor fixed to 0.5 and second mutation operator. The SA is also applied to solve this problem. To verify the performance of the proposed algorithm, this case was repeatedly solved one hundred times. The best and worst values among the best solutions of these one hundred runs are listed in Table. III. The average value and standard deviation (STD) for the best solutions of these one hundred runs is shown in this table. The best solutions for these one hundred runs are compared with the best objective function value obtained by the simulated annealing. The Exhaustive Search method is also repeatedly used to solve this problem one hundred times. The best function value obtained by the Exhaustive Search is $\$ 118,538.53$ / year. The best function value obtained by the Exhaustive Search is the same as that obtained by the CODEQ.

Case 2: All buses are available for placement of capacitors. Parameters for the CODEQ application are selected as those of case 1 , except that the maximum generation is set to 5000. The $\mathrm{DE}$ and SA are also applied to solve this problem. Table IV expresses the best and the worst values among the best solutions of one hundred runs. The average value and standard deviation for the best solutions of those one hundred runs are also listed in this table. From the computational results, it is observed that the SA method cannot find the global solution in one hundred runs. The worst objective function value of these one hundred runs obtained by the CODEQ method is smaller than those of the DE and SA. Comparing the results of the DE and CODEQ, reveals that they are almost the same. However, the performance of DE is dependent on the choice of the mutation operator. The best and average results of CODEQ are relatively better than those of the other three methods. The best objective function value of these one hundred runs is $\$ 115,398.17$ / year.

\section{CONCLUSION}

Several heuristic methods including the CODEQ, DE, and SA used to solve one benchmark function and one capacitor placement problem had been described in this work. The concepts of chaotic search, opposition-based learning, and quantum mechanics are used in the CODEQ method to
TABLE I

COMPUTATION RESULTS FOR ONE HUNDRED RUNS OF EXAMPLE 1

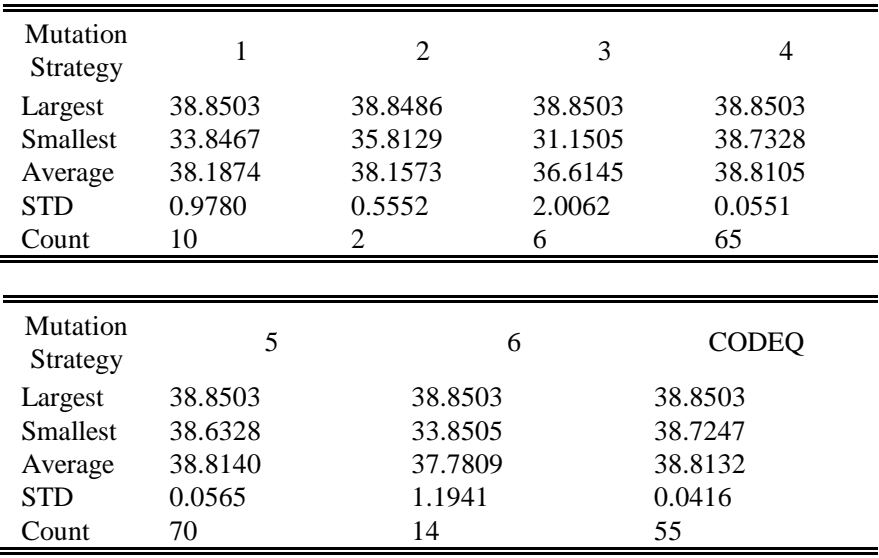

TABLE II

STANDARD DEVIATION FOR VARIOUS POPULATION SIZE

\begin{tabular}{lrrrr}
\hline \hline $\begin{array}{c}\text { Population } \\
\text { size }\end{array}$ & 1 & 2 & 3 & 4 \\
10 & 1.7443 & 1.1668 & 3.1256 & 0.4549 \\
5 & 2.6685 & 2.0677 & 3.5187 & 1.2180 \\
\hline \hline \multicolumn{2}{c}{5} & & 6 & CODEQ \\
\hline \hline Population & size & & 6 & 0.1346 \\
10 & 0.9143 & 1.7201 & 0.4363 \\
5 & 1.2985 & 2.9524 &
\end{tabular}

TABLE III

THE COMPUTATION RESULTS OF CASE 1 FOR EXAMPLE 2

\begin{tabular}{llll}
\hline \hline Method & CODEQ & DE & SA \\
& & & \\
Best & $118,538.53$ & $118,538.53$ & $118,763.64$ \\
Worst & $118,763.64$ & $130,300.02$ & $125,326.81$ \\
Average & $118,540.78$ & $119,811.67$ & $123,373.86$ \\
STD & 22.51 & $1,944.05$ & $1,999.16$ \\
\hline \hline
\end{tabular}

overcome the drawback of selection of the crossover factor, scaling factor, and mutation operator used in the original differential evolution (DE) method. From example 1, the convergence property of the CODEQ method is outperforming than the original DE method. From example 2, the computation results shows that the solution obtained by the CODEQ method is better than those obtained by the DE and SA methods.

\section{ACKNOWLEDGMENT}

Financial research support from the National Science Council of the R. O. C. under grant NSC 100-2632-E-131-001-MY3 3/3 is greatly appreciated.

\section{REFERENCES}

[1] H. D. Chiang, J. C. Wang, O. Cockings, and H. D. Shin, "Optimal capacitor placements in distribution systems: Part1: A new formulation and the overall problem," IEEE Trans. on Power Delivery, vol. 5, pp. 634-642, 1990 .

[2] H. D. Chiang, J. C. Wang, O. Cockings, and H. D. Shin, "Optimal capacitor placements in distribution systems: Part2: Solution algorithms 
and numerical results," IEEE Trans. on Power Delivery, vol. 5, pp. 643-649, 1990.

[3] J. J. Grainger and S. H. Lee, "Optimum size and location of shunt capacitors for reduction of losses on distribution feeders," IEEE Trans. on Power Apparatus and Systems, vol.100, no. 1, pp. 1105-1108, 1981.

[4] S. H. Lee, and J. J. Grainger, "Optimum placement of fixed and switched capacitors on primary distribution feeders," IEEE Trans. on Power Apparatus and Systems, vol. 100, pp. 345-352, 1981

[5] J. J. Grainger, and S. H. Lee, "Capacity release by shunt capacitor placement on distribution feeders: A new voltage-dependent model," IEEE Trans. on Power Apparatus and Systems, vol. 101, pp.1236-1244, 1982.

[6] J. L. Bala, P. A. Kuntz, and M. Tayor, "Sensitivity-based optimal capacitor placement on a radial distribution feeder," in Proc. Northcon 95 IEEE Technical Application Conf., pp. 225-230, 1995.

[7] S. Sundhararajan and A. Pahwa, "Optimal selection of capacitors for radial distribution systems using a genetic algorithm," IEEE Trans. on Power Systems, vol. 9, pp. 1499-1507, 1994.

[8] R. Storn and K. V. Price, "Minimizing The Real Functions of the ICEC '96 Contest by Differential Evolution," IEEE Conference on Evolutionary Computation, pp.842-844, 1996.

[9] S. Sultana and P. K. Roy, "Optimal capacitor placement in radial distribution systems using teaching learning based optimization," International Journal of Electrical Power and Energy Systems, vol.54, pp.387-398, 2014.

[10] R. Muthu Kumar and K. Thanushkodi, "Reconfiguration and capacitor placement using opposition based differential evolution algorithm inpower distribution system," International Review on Modelling and Simulations, vol. 6, no. 4, pp. 1233-1239, 2013

[11] J. Olamaei, M. Moradi, and T. Kaboodi, "A new adaptive modified firefly algorithm to solve optimal capacitor placement problem," $18^{\text {th }}$ Electric Power Disteibution Network conference, art. No. 6565962, 2013.

[12] K. V. Price, "Differential Evolution vs. Functions of the $2^{\text {nd }}$ ICEC," IEEE Conference on Evolutionary Computation, pp.153-157, 1997.

[13] Y. C. Lin, K. S. Hwang, and F. S. Wang, "Plant scheduling and planning using mixed-integer hybrid differential evolution with multiplier updating," Congress on Evolutionary Computation, San Dego, CA., pp.593-600, 2000

[14] M. G. H. Omran and A. Salman, "Constrained Optimization Using CODEQ," Chaos, Solitons and Fractals, vol. 42, pp.662-668, 2009.

[15] M.G.H. Omran and A. Salman, "Improving the performance of CODEQ using quadratic interpolation," $2^{\text {nd }}$ International Conference on Agents and Artidicial Intelligence Proceedings, vol. 1, pp. 265-270, 2010.

[16] M.G.H. Omran and A. Salman, "Optimization of discrete values using recent variants of differential evolution," Proceedings of IASTED International Conference on Computational Intelligence, pp. 108-113, 2009.

[17] C. T. Su, C. S. Lee, and C. S. Ho, "Optimal selection of capacitors in distribution systems," in Proc. IEEE Power Tech. Conf., BPT99-171-42, 1999.

[18] J. Sun, B. Feng, and W. Xu, "Particle swarm optimization with particles having quantum behavior," IEEE Congress on Evolutionary Computation, pp.325-331, 2004.

[19] B. Goffe, "Global optimization of statistical functions with simulated annealing," Journal of Economics, vol. 60, pp. 65-100, 1994.

[20] Z. Michalewicz, "Genetic algorithms + data structures = evolution programs". New York: Springer-Verlag, 2012.
Ji-Pyng Chiou, (1966) received his Ph. D. degree in Electrical Engineering from National Chung Cheng University, Chiayi, Taiwan. He is currently a Professor of the Department of Electrical Engineering at Ming Chi University of Technology, New Taipei City, Taiwan. His research interest is in the application of evolutionary algorithms and optimization techniques.

Chung-Fu Chang, (1964) received his Ph. D. degree in Electrical Engineering from National Chung Cheng University, Chiayi, Taiwan. $\mathrm{He}$ is currently an Associate Professor of the Department of Electrical Engineering at WuFeng University, Chiayi City, Taiwan. His field of interest includes distribution system planning, reliability engineering, optimization techniques, and $\mathrm{AI}$ application. 